\title{
Brain Space for Learned Song in Birds Develops Independently of Song Learning
}

\author{
Eliot A. Brenowitz, ${ }^{1}$ Karin Lent, ${ }^{1}$ and Donald E. Kroodsma ${ }^{2}$ \\ ${ }^{1}$ Departments of Psychology and Zoology, University of Washington, Seattle, Washington 98195 and ${ }^{2}$ Department \\ of Biology, University of Massachusetts, Amherst, Massachusetts 01003
}

In numerous species of birds, individuals or species that sing larger numbers of song types have larger song control nuclel In thelr bralns. The direction of the cause and effect relationship between the complexity of song behavior and brain space is unknown, however. The hypothesis that birds that learn large song repertoires develop large song nuclei was therefore tested with a songbird, the marsh wren (Cistothorus palustris). Males were hand-reared and tutored in the laboratory with either small ( $n=8$ males heard 5 song types) or large ( $n=8$ males heard 45 song types) repertoires. When the birds were adults, the number of song types each male sang was first determined, and then the volume and certain cellular attributes of the song nuclei HVC and RA were measured. The two groups of wrens showed large behavioral differences in the size of their learned song repertoires, but did not differ in either the volumes of HVC and RA or in neuronal size, number, or density within these nuclei. These results suggest that the relatlonship between behavioral song complexity and brain space found in this and other species develops largely independently of early song learning experience and the later production of those songs.

[Key words: bird, birdsong, brain space, development, learning, marsh wren, song, songbird]

Song is a learned behavior that is regulated by a discrete network of brain nuclei in songbirds. Individuals or species with more complex singing behavior often have larger song nuclci devoted to controlling this behavior (e.g., Nottebohm et al., 1981; Canady et al., 1984; Kroodsma and Canady, 1985; Brenowitz and Arnold, 1986; Brenowitz et al., 1993; DeVoogd et al., 1993). Furthermore, neuronal size, number, and spacing within the song control nuclei are also greater in birds with larger repertoires (Brenowitz et al., 1993). This correlation between behavioral complexity and brain characteristics has received much attention as a possible example of structural changes in a vertebrate brain associated with processes of learning and memory (e.g., Bailey and Kandel, 1993).

\footnotetext{
Received Jan. 30, 1995; revised May 4, 1995; accepted May 24, 1995
}

We thank the following people for their assistance: Melissa Kroodsma, for hand-rearing the marsh wrens; Stacy Malone, Ben Mullaly, Kathy Iennaro, Patti Fallon, Elena Murgu, and especially Judy Wells, who sorted songs and established song repertoires; Christine Collins for perfusing the birds; and Belinda Nalls for help with histology. This research was supported by NSF Grant BNS-9111666 to D.E.K., and NSF Grant IBN 9120540 and an Alfred P. Sloan research fellowship to E.A.B.

Correspondence should be addressed to Eliot A. Brenowitz, Department of Psychology, Box 351525, University of Washington, Seattle, WA 98195.

Copyright $(\mathcal{C} 1995$ Society for Neuroscience $0270-6474 / 95 / 156281-06 \$ 05.00 / 0$
Within a given species, the number of song types (i.e., the song repertoire) and the size of song nuclei may be positively correlated, but the direction of the cause and effect relationship between these variables, if any, is unknown. Three hypotheses can be proposed. (1) Birds that learn large song repertoires could, as a consequence, develop larger song nuclei. (2) Birds endowed with larger song nuclei might be enabled subsequently to learn larger numbers of song types. (3) Both the size of song nuclei and the number of songs learned may be regulated independently but in parallel by one or more other factors, either genetic, epigenetic, or environmental in nature (see also Nottebohm et al., 1981; Arnold, 1992). The first hypothesis can be distinguished from the other two by the predicted effects on brain space of manipulations of early song learning experience. If the size of song nuclei is determined by the complexity of song learning, then one should be able to guide the development of song nuclei in predictable directions by controlling the amount of song learned by individuals. According to the last two hypotheses, however, manipulations of song learning should not have a predictable effect on the size of song nuclei.

Our study tested the prediction of the first hypothesis, that manipulations of song learning experience should have consistent effects on the size of song nuclei. We hand-reared marsh wrens (Cistothorus palustris) and assigned males to groups that were tutored with either small or large song repertoires. Previous studies demonstrated a correlation between individual song complexity and brain space in this species (Canady et al., 1984; Kroodsma and Canady, 1985). Having successfully created two groups with large differences in song repertoire size, we then searched for differences in quantitative structural attributes of the song nuclei between the groups. We found no neuroanatomical differences between the large and small repertoire groups and therefore we could falsify the hypothesis that the complexity of learned song behaviors has a major influence on the brain characteristics that we measured. The lack of brain differences, despite large behavioral differences, suggests that previous reports of correlations between repertoire size and brain space in this species are a result of birds with well-endowed brains being enabled to sing larger repertoires, or that both brain space and song repertoire size develop under the influence of one or more other factors.

\section{Materials and Methods}

Subjects and song tutoring routine. The nestling marsh wrens for this study $(n=16)$, about $8-12 \mathrm{~d}$ old, were collected between 28 June and 8 July 1992 in marshes at the mouth of the Housatonic River in Connecticut and near Tivoli on the Hudson River in Ncw York (federal permit, PRT-672911; CT permit, no number; NY permit, LCP92-307). The birds were reared in the laboratory on a modified Lanyon diet 
(Lanyon, 1979). As birds fledged at about $14 \mathrm{~d}$ of age, males were identified by their heavier weights and by subsong and were placed into four treatment groups, each of which was destined to hear a different tutor tape. During the hand-rearing period, two to three males were kept in each cage.

Four tutor tapes were prepared from a repertoire of 50 song types recorded from a single male marsh wren on the Housatonic River in Connecticut. Mean song repertoire size for eastern marsh wrens is ca. 54 song types (Canady et al., 1984). The two small repertoire tutor tapes each contained 5 song types, and the two large repertoire tutor tapes each contained 45 song types. Each tape consisted of a continuous sequential presentation of the 5 or 45 song types on the tape over a 51 min period, at a rate of about 13 songs/min, so that each male was exposed to the same quantity, but not quality, of tutoring. Tutoring began on 11 July, when the males were $18-25 \mathrm{~d}$ old. Two small and two large repertoire tutoring groups were established, with siblings distributed among groups, and with four males in each group. All birds were reared in the same room, so for tutoring the appropriate cages were carried to another room and songs broadcast, over a large speaker placed next to the cages, for either 45 or $90 \mathrm{~min}$ on a given day.

After all the birds were feeding on their own, they were moved on 3 August from the single-room nursery to the animal rooms in the Morrill Science Center at the University of Massachusetts. There, each male was placed into his own wooden cage with a wire "canary front" and the four groups were isolated from each other in different rooms. With cages placed side by side, males could hear but not see each other. A speaker was hung on the front of each cage, and males were tutored through August. Over a period of about $50 \mathrm{~d}$, then, from 11 July through August, each male heard about 33,000 songs over the speakers. Those tutored with 5 song types heard each type about 6600 times, and those tutored with 45 types heard each about 750 times.

During the spring of 1993, the tutor tapes were again played to the males. Although we have little evidence for learning from tapes in the spring (Kroodsma and Pickert, 1980), we wanted to take no chances and wanted to reinforec to the males the repertoires that they were to learn. Males in the small repertoire group might have improvised more songs, for example, if we had not presented them with more examples of the repertoires to which we wanted to limit them.

At the University, male marsh wrens were housed, by necessity, with songbirds of several other species. Previous studies of song ontogeny in marsh wrens had revealed little evidence of interspecific vocal learning (e.g., Kroodsma and Pickert, 1984), but we nevertheless tried to balance the quantity and quality of interspecific auditory experience among the wrens. All wren males thus heard four whistled songs of black-capped chickadees (Parus atricapillus). Also, marsh wrens in all tutor groups heard equal quantities of gray catbird (Dumetella caroli. nensis) song each day in August. Males in the two small repertoire groups heard a repeated $5 \mathrm{sec}$ segment for about $45 \mathrm{~min}$ each day, and Indes in the two large repertoire groups heard a repeated 16 min segment for the same time. We thus minimized the interspecific auditory experience for birds in the small repertoire group, because we felt it might be necessary to prevent song improvisation with such a deficient intraspecific experience of only 5 song types. As expected, when the males sang in 1993, we found no evidence of interspecific vocal learning. We therefore do not believe that these interspecific sounds were a factor in our experiments.

During the hand-rearing phase and through September 1992, the wrens were maintained on a $15 \mathrm{hr}$ day length. During October and November, daylengths were gradually reduced from 15 to $11 \mathrm{hr}$. After 8 weeks on short days, daylengths were increased gradually from 28 January to 23 February to $15.5 \mathrm{hr}$, where they were maintained through the end of the experiment.

For part of another experiment, it was necessary to expose males in both tutoring groups to each other. Before such exposure, however, we verified that each male's song development was completed, and that the male was in full, adult, stable song. If song development had not been completed, we would have risked pronounced alteration of repertoires as males learned additional song types from each other. This risk may have been most pronounced for males in the small repertoire tutor group. We therefore carefully monitored the song development of the males, and judged the quality of the singing every few days during March. When we could detect no instability in a male's song for a period of about a week, we considered his song development complete, and then felt we could safely expose each male to singing by the males in the other tutor group. These interactive experiments were completed between 20 March and 10 April, after which the males were again isolated in their own tutor groups. We examined about 500 songs recorded from four males in the small repertoire group after the interactive experiments, and compared these songs with the repertoires of the same males recorded before the interactive studies. Of the 500 postinteractive study songs, only five songs did not obviously match one of the original five to six song types found in the preinteractive study repertoires of the four males. These five songs were unstable and reminiscent of song produced during the plastic stage of song development. Such unstable songs are not atypical for any bird singing under laboratory conditions. We conclude from these results that the males had achieved stable, adult song repertoires before birds in the two tutoring groups were exposed to each ohler.

We used two basic techniques to determine the song repertoires of the male marsh wrens. For males in the small repertoire tutor group, we monitored their limited repertoires on the screen of a Kay Elemetrics DSP 5500 Sona-graph. New song types were printed, and a catalog of song types for each male was quickly established. When a long string of songs occurred with no new types observed, we considered the catalog completed; male 1 thus sang 6 different song types in his first 27 songs, but no new types were found in the next 120 songs. Repertoires of males tutored with 45 song types were much larger and could not be readily determined by visual inspection on the DSP 5500 screen. We therefore printed 100-200 songs from each of these males and sorted the sonagrams into different types. An unbiased observer (J. Wells) inspected the sonagrams to ensure that the same standards were maintained among all the males. Io estimate the repertoire size based on our sample, we used an estimate of sample coverage (see Canady et al., 1984). Briefly, the method is as follows. First, we determined the number of independent occurrences of each song type, which is typically less than the total number of printed songs. Because marsh wrens tend to repeat several renditions of a song type in a bout of singing, we dictated that successive renditions of a given song type must be separated by at least 10 songs of other types to score those successive renditions as being independent. Next, the number of song types that occurred only once in the sample was determined. Sample coverage, which is the probability that the next song sampled will be of a type already sampled, is then $1-S / N$, where $S$ is the number of types occurring only once and $N$ is the total number of independent occurrences of all song types in the sample. The observed repertoire size divided by the sample coverage then yields an estimate of actual repertoire size. In our samples, repertoires were adjusted by $0-5$ song types based on this approach.

Analysis of song nuclei. When they were approximately 10 months old, all 16 birds were perfused with heparinized phosphate buffer $(\mathrm{pH}$ 7.4) followed by $4 \%$ paraformaldehyde on 24 and 25 April, 1993. The brains were removed and placed in the fixative for at least 2 weeks. Either the right or left lobe of the telencephalon was separated from the brain of each bird. The intact telencephalic lobe was used in the present study, and we balanced the left and right lobes between the small and large repertoire tutor groups. The other, detached telencephalic lobe from each bird was used in a companion study of dendritic arborizations of neurons in song nuclei (Airey et al., 1994). A previous study of wrens from this same population in Connecticut did not detect any consistent differences in the sizes of telencephalic song nuclei between the left and right sides of the brain (Brenowitz et al., 1993).

We embedded the intact part of the brain in gelatin. The gelatin block was trimmed and placed in a $20 \%$ sucrose/formalin solution for $48 \mathrm{hr}$. Transverse frozen sections were cut serially at a thickness of $50 \mu \mathrm{m}$ and collected into avian saline. Alternate sections were nounted and stained with thionin.

Using these Nissl-stained sections, we measured the volumes of two song nuclei, the high vocal center (HVC) and the robust nucleus of the archistriatum (RA), and the volume of a visual region, the rotund nucleus (Rt), in the thalamus (see Table 1 for sample sizes). Our measures of HVC included the caudal-most portion, which extends medially and ventrally along the ventricle and contains large, densely packed cells. Rt was measured as a control nucleus because it is not part of the song system, and its borders are well defined in Nissl-stained tissue. A magnified image of each alternate section was projected on paper at $47 \times$, the perimeter of the relevant nucleus was traced, and the area was calculated with a digitizing tablet and microcomputer. To obtain the total volume for each nucleus, we multiplied the area of each section by the sampling interval of $100 \mu \mathrm{m}$ and summed the volumes of all individual sections taken through the nucleus on one side of the brain for HVC 
Table 1. Lack of significant differences in anatomical measurements $(X \pm S E M)$ in brains of marsh wrens tutored with a small song repertoire (five song types) or a large song repertoire (45 song types)

\begin{tabular}{lrrrl} 
& \multicolumn{1}{c}{ Small rep } & \multicolumn{1}{c}{ Large rep } & df & $P$ \\
\hline HVC $\left(\times 10^{-1} \mathrm{~mm}^{3}\right)$ & $5.03 \pm 0.04$ & $5.32 \pm 0.05$ & 14 & 0.68 \\
HVC/Rt $\left(\times 10^{-1}\right)$ & $5.33 \pm 0.04$ & $5.67 \pm 0.75$ & 12 & 0.70 \\
HVC/telencephalon $\left(\times 10^{-3}\right)$ & $3.51 \pm 0.53$ & $4.25 \pm 0.44$ & 12 & 0.30 \\
HVC somal size $\left(\mu \mathrm{m}^{2}\right)$ & $97.2 \pm 4.2$ & $103.2 \pm 3.70$ & 8 & 0.31 \\
HVC neuronal density & & & & \\
$\quad\left(\times 10^{5}\right.$ neurons $\left./ \mathrm{mm}^{3}\right)$ & $1.85 \pm 0.15$ & $1.70 \pm 0.39$ & 8 & 0.73 \\
HVC neuron no. $\left(\times 10^{4}\right)$ & $9.19 \pm 0.49$ & $8.87 \pm 0.72$ & 8 & 0.72 \\
RA $\left(\times 10^{-1}\right.$ mm & $1.52 \pm 0.01$ & $1.70 \pm 0.02$ & 14 & 0.40 \\
RA/Rt $\left(\times 10^{-1}\right)$ & $2.14 \pm 0.40$ & $2.19 \pm 0.40$ & 12 & 0.93 \\
RA/telencephalon $\left(\times 10^{-3}\right)$ & $2.44+0.13$ & $2.72+0.29$ & 12 & 0.40 \\
RA somal size $\left(\mu \mathrm{m}^{2}\right)$ & $149.80 \pm 5.06$ & $163.92 \pm 5.28$ & 8 & 0.09 \\
RA neuronal density & & & & \\
$\quad\left(\times 10^{4}\right.$ neurons $\left./ \mathrm{mm}^{3}\right)$ & $5.16 \pm 0.67$ & $4.57 \pm 0.59$ & 8 & 0.18 \\
RA neuron no. $\left(\times 10^{3}\right)$ & $7.55 \pm 0.51$ & $8.72 \pm 1.21$ & 8 & 0.61 \\
Rt $($ mm $)$ & $0.95 \pm 0.05$ & $0.94 \pm 0.05$ & 14 & 0.84 \\
Rt/telencephalon $\left(\times 10^{-3}\right)$ & $7.62 \pm 0.44$ & $7.65 \pm 0.25$ & 12 & 0.95 \\
Telencephalon $\left(\mathrm{mm}^{3}\right)$ & $126.08 \pm 1.88$ & $126.52 \pm 3.18$ & 12 & 0.91
\end{tabular}

Degrees of freedom (df $=n_{1}+n_{2}-2$ ) and probability levels $(P)$ for $t$ tests (two-tailed) are indicated for each anatomical attribute.

RA, and Rt. The observer was blind to the size of the tutored song repertoire for this measurement and all others.

To control for possible differences between the small and large tutor repertoire groups in factors such as body size, overall brain size, or histological preparation, we also compared the volumes of $\mathrm{HVC}$ and $\mathrm{RA}$ relative to the volumes of $\mathrm{Rt}$ and of the total telencephalon on that side of the brain (see Brenowitz et al., 1993, for rationale). The total volume of the telencephalon was estimated using the same methods as in our previous study of marsh wrens (Brenowitz et al., 1993; see also DeVoogd et al., 1993). In the rostral- and caudal-most extents of the telencephalon, the entire hemispheric lobe was traced. In sections where the diencephalon was contiguous with the telencephalon, the septomesencephalic tract ( $\operatorname{TrSM})$, anterior commisure (CoA), and occipitomesencephalic tract $(\mathrm{OM})$ were used as natural borders of the telencephalon. For comparable use of nonsong nuclei such as Rt to normalize the volumes of song nuclei, see Nottebohm (1981) and Simpson and Vicario (1991).

We also measured cellular attributes of RA and HVC, including neuron density, neuron number, and the area of neuronal somata, for five males arbitrarily selected from each sized tutor group. Cells with a relatively large soma, with a clear nucleus generally containing only one large, darkly staining nucleolus, and with a uniform nongranular cytoplasm, were judged to be neurons. Neuronal density was measured through microscope optics at a magnification of $1250 \times$, using the methods described below. Somal area was measured with a calibrated image analysis system (Imaging Research, Inc.).

We used a randomized sampling scheme to measure neuronal density and somal area throughout RA and HVC. Initially we did a preliminary analysis to determine the minimum number of neurons that had to be sampled to achieve the maximum variance around the mean for these cellular attributes in each nucleus. This minimum sample size differed for RA (100 neurons) and HVC (140 neurons) because of greater variance in cell size in HVC.

$R A$ neuron sampling. Before our analyses, we observed an arbitrarily selected slide from each sized tutor group under a $100 \times$ objective to estimate the average number of cells in a single visual field on the video monitor. We used this number to determine the total number of visual fields to be analyzed to achieve the minimum sample of 100 neurons. The number of different visual fields analyzed per bird ranged from 24 to 33 , and these were distributed among all the sections ascertained to contain RA. Under a $10 \times$ microscope objective, the dorsal, ventral, medial, and lateral borders of RA in each section were labeled on the coverslip with a marking pen to provide landmarks to guide the cellular analysis under a $100 \times$ objective. Using the $100 \mathrm{X}$ objective, we deter- mined the number of visual video fields required to span the full extent of RA in each section, and then alternated between dorsal-ventral and medial-lateral transects in successive sections. A random number generator was used to determine which of these fields to analyze on each axis. We analyzed 2-7 video fields per section, with an average of 34 fields, depending on the size of RA in the sampled section.

For each sampled field we traced with a computer mouse the perimeters of all individual neuronal somata observed on screen at a total magnification of $312.5 \times$. The area of each outlined somata was determined automatically by the imaging system. In the same field we determined neuronal density by counting the number of neuronal nucleoli in a sample volume of $8.959 \times 10^{-5} \mathrm{~mm}^{3}$. Nucleoli were small relative to the section thickness of $50 \mu \mathrm{m}$, and we therefore made no correction for nucleolar splitting (Cammermeyer, 1967; Konigsmark, 1970).

We estimated the total number of neurons in RA by multiplying mean neuronal density by the volume of RA for each bird. This procedure assumes that neuronal density is uniform throughout the extent of RA. We tested this assumption by determining for each bird whether density varied as a function of location in RA along the rostral-caudal axis. Visual inspection of mean neuronal density in each section plotted against the location of the sample in RA indicated that the relationship between these two variables was best approximated by a linear model. Linear regression analysis indicated, however, that for each bird the relationship between neuronal density and rostral-caudal location in RA was not significant $\left(r^{2} \leq 0.06 ; P=0.17\right.$ for one bird, and $P \geq 0.45$ for all other birds). This pattern of results indicates, then, that neuronal density is uniform throughout RA.

Determining the mean neuronal somata area for RA as a whole similarly assumes a uniform distribution of cell size throughout the nucleus. We also tested this assumption with linear regression analyses. We first determined for each bird the mean somatal area in each section as a function of sample location in RA along the rostral-caudal axis. For all five birds that we tested in the small repertoire tutor group, and for three of the five tested birds in the large repertoire tutor group, we found no significant relationship between somatal area and rostral-caudal location in RA ( $r^{2} \leq 0.39 ; P=0.06$ for one bird from each tutor group, $P \geq 0.14$ for remaining birds). Two of the five birds in the large repertoire tutor group did show a significant relationship between these variables $\left(r^{2}=0.66\right.$ and $0.75, P=0.05$ and 0.03 , respectively). A Fisher exact probability test indicated, however, that one could expect this pattern of test results by chance alone $(P=0.444$, two-tailed). We therefore concluded that the size of neuronal somata did not vary consistently with location in RA.

$H V C$ neuron sampling. HVC sampling was done with the same ra- 
tionale, but we modified our technique to accommodate HVC's irregular shape, greater volume, and greater heterogeneity in neuronal size compared to RA. Starting with the most rostral section to contain HVC, we sampled neuronal density and somatal area in a repeating sequence of two out of every three successive sections throughout the nucleus. The ventral and lateral extents of HVC were marked on the coverslip as described above; the dorsal and medial limits of this nucleus are clearly delineated by the ventricular zone along the lateral ventricle. In each sampled section, we counted the number of visual video fields that spanned HVC in the medial-lateral axis. A random number generator was used to determine the column(s) along the medial-lateral transect from which the samples would be taken. For each selected column, we then measured the number of visual video fields that spanned the dorsalventral axis of $\mathrm{HVC}$ at that point. Using a random number generator to determine which field to sample on the dorsal-ventral transect, we then analyzed 2-5 video fields per section, with an average of 4-5 fields, depending upon the size of HVC in the sampled section. Neuronal density and somatal area were measured at each selected video field as described for RA. Using this scheme we sampled $\geq 140$ cells for each bird.

As for RA, we used a linear regression analysis to determine whether neuronal density was uniform along the rostral-caudal axis of HVC in each bird. For all five birds in the small repertoire tutor group, and for four out of five birds in the large repertoirc tutor group, we found no significant relationship between neuronal density and rostral-caudal location $\left(r^{2} \leq 0.12 ; P=0.09\right.$ for one bird in the small repertoire group, $P \geq 0.28$ for other birds). For one bird in the large repertoire tutor group, we found a significant relationship between these variables $\left(r^{2}\right.$ $=0.30, P=0.003$ ), but this pattern of test results could have arisen by chance (Fisher exact probability test, $P=1.00$, two-tailed).

To determine whether neuronal density was also uniform along the medial-lateral axis of $\mathrm{HVC}$, we compared mean densities in the medial and lateral portions of the nucleus with a $t$ test. In the rostral- and caudal-most regions of $\mathrm{HVC}$, where the nucleus has the smallest crosssectional area, we collected a maximum of two samples in single sections. This sampling scheme precluded a regression analysis of data collected along the medial-lateral axis. The judgement of whether a sample was collected from the medial or lateral "halves" of IIVC was based on the location of each sampled video field relative to the number of fields that spanned the nucleus on this axis. For all birds in the large repertoire tutor group, and for three birds in the small repertoire group, the mean neuronal density in the medial and lateral portions of HVC was not significantly different $(P=0.065$ for one bird, $P \geq 0.34$ for other birds). In two birds in the small repertoire group, neuronal density was significantly greater in the medial portion of HVC $(P=0.016$ and 0.006 ), but again this distribution of test results could have arisen by chance (Fisher exact probability test, $P=0.444$, two-tailed). From these results we concluded that neuronal density was sufficiently uniform throughout HVC to justify using a single mean measurement for the entire nucleus of each bird in intergroup comparisons. Consequently, we estimated the total number of neurons in each bird's HVC by multiplying mean neuronal density by the volume of HVC.

We used linear regressions for each bird to test whether mean neuronal somatal area was uniform throughout HVC along the rostral-caudal axis. For all birds in both tutor groups, somatal area and rostralcaudal location were not significantly related $\left(r^{2} \leq 0.42 ; P=0.08\right.$ for one bird, $P \geq 0.15$ for other birds). We also determined whether somatal area was uniform along the medial-lateral axis of HVC in the same way as described above for neuronal density. For all birds in both tutor groups, the mean somal areas of neurons in the medial and lateral portions of HVC were not significantly different $(P=0.08$ for one bird, $P \geq 0.12$ for other birds). From these results we concluded that the area of neuronal somata was sufficiently uniform throughout HVC to justify using a single mean measurement for the entire nucleus of each bird.

\section{Results}

Song hehavior. The song repertoires of the males in hoth the small and large repertoire tutor groups were clearly determined, to a large extent, by their early tutoring experience (Fig 1). Repertoires of all eight males in the small repertoire tutoring group were restricted to five or six song types, all of which tended to be good copies of the five tutor songs or otherwise normal marsh wren songs. Males in the large repertoire tutor group were also
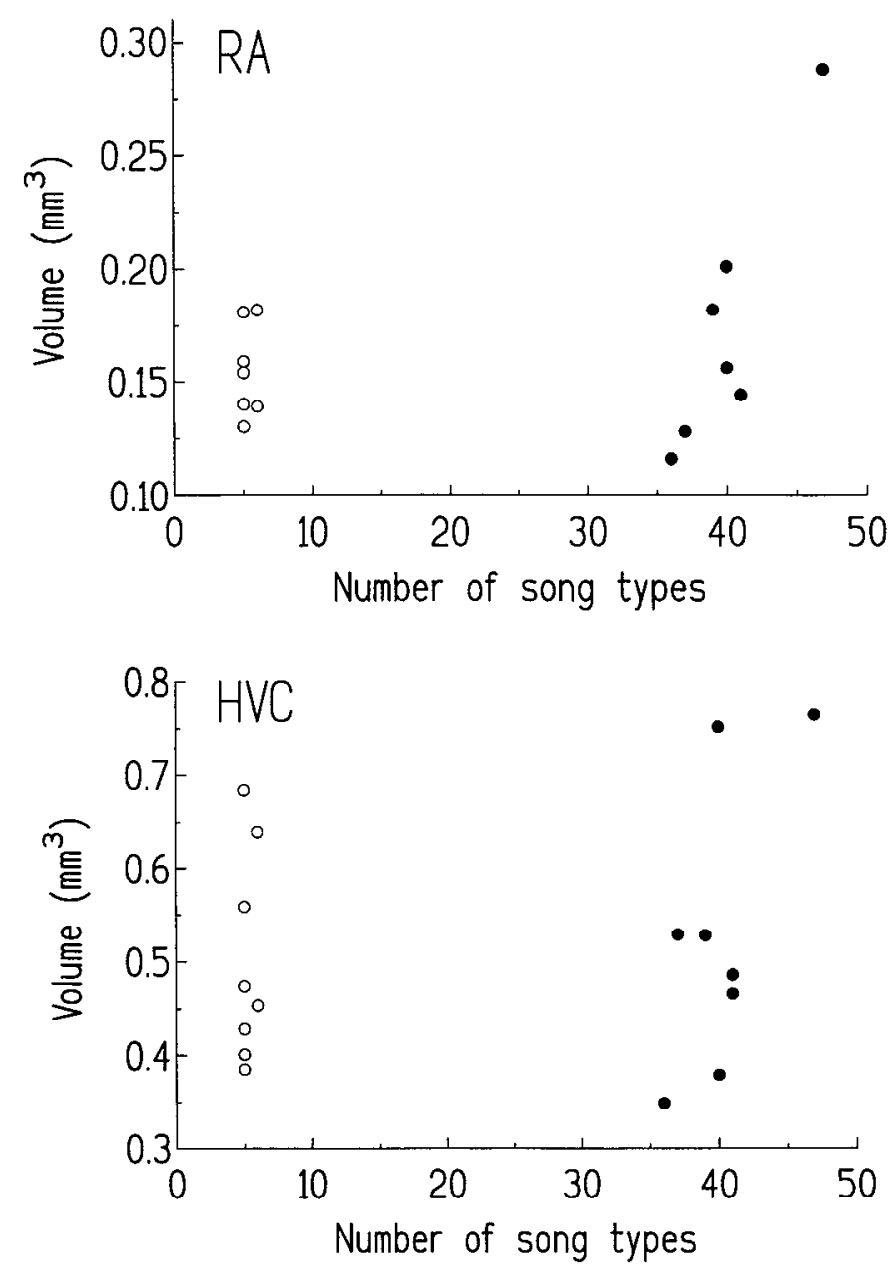

Figure 1. The number of song types learned by male marsh wrens does not influence the volume of two song control nuclei, RA (top) and HVC (bottom) (see Table 1 for tests). Within the large repertoire group (solid circles), the volume of RA was significantly correlated with repertoire size $(r=0.86, P=0.006)$ and the volume of HVC approached significance $(r=0.63, P=0.09)$. Neillier volume was related to repertoire size in the wrens with the small repertoires (open circles) (RA: $r=0.25, P=0.54$; HVC: $r=0.24, P=0.56$ ).

clearly influenced by the 45 song types on their tutor tapes, and developed repertoires of $36-47$ (median $=41$ ) normal-sounding marsh wren songs.

Song nuclei. Despite the striking difference in behavioral repertoire size between the two tutor groups, the brain space devoted to producing small and large repertoires did not differ. The unilateral absolute volumes of either RA or HVC did not differ between birds in the small and large repertoire tutor groups (Fig. 1, Table 1). The variance around the mean differed significantly between the two tutor groups for the absolute volume of RA $(F=4.00$, df $=7,7, P<0.05)$, but not for HVC $(F=1.56, \mathrm{df}=7,7, P>0.05)$. The volumes of RA and IIVC relative to the volumes of either $\mathrm{Rt}$ or the entire telencephalon also did not differ between the different sized tutor groups (Table 1).

Within the two tutoring groups, the relationship between song repertoire size and brain space devoted to song differed. The number of song types produced by birds in the small repertoire tutor group did not correlate with the absolute volume of either RA or HVC (see Fig. 1 caption), as might be expected given 
the low range of repertoire sizes in this group. In the large repertoire tutor group, however, the volume of RA was significantly correlated with song repertoire size, and the correlation between $\mathrm{HVC}$ volume and song repertoire size approached significance (see Fig. 1 caption).

The pattern of cellular attributes of the song nuclei in the two tutor groups paralleled that found in the volume of these nuclei. We found no differences in the somal area, density, and number of neurons in RA and HVC between the small and large repertoire tutor groups (Table 1).

\section{Discussion}

Our song tutoring paradigm successfully resulted in two groups of birds with pronounced differences in the numbers of song types in adult repertoires. In nature, song repertoires of individual males in these eastern marsh wren populations range from about 40-70 song types (e.g., Canady et al,, 1984). In the socially impoverished conditions of the laboratory, however, males do not learn repertoires as large as those that may occur in nature. Male wrens tutored in the laboratory with 200 song types, for example, learned to produce a median of 45 song types (Kroodsma and Canady, 1985), which is quite similar to the median of 41 song types in our large repertoire tutor group. In previous experiments (Kroodsma, 1979, unpublished data), males had successfully been held to repertoires of six to nine song types, and data here further verify that development of a song repertoire can be determined to a large extent by experience. These small repertoires are well below the minimum number of song types produced by wild birds (Canady et al., 1984).

Despite the large differences in the adult song repertoires of our two experimental groups, however, we found no group differences in either the absolute or relative amounts of brain space devoted to song, or in cellular attributes that we measured in these song nuclei. Our results indicate that birds can have large song nuclei but small song repertoires if the males experience impoverished auditory stimulation early in life (see also Nottebohm et al., 1981; Arnold, 1992). This observation explains the lack of correlation between song repertoire size and the volume of song nuclei found within the small repertoire tutor group. Males in the large repertoire group were tutored with as many song types as they might have been expected to learn in the laboratory (Kroodsma and Canady, 1985). The relationship between repertoire size and the volume of song nuclei within this large repertoire group therefore simulates the conditions found in nature and in laboratory experiments in which a relationship between brain space and repertoire size has been found (Nottebohm et al., 1981; Canlady et al., 1984; Kroodsma and Canady, 1985; Brenowitz and Arnold, 1986; Brenowitz et al., 1993; DeVoogd et al., 1993).

The results of this study therefore support the null hypothesis that we tested, that is, that the complexity of learned song behaviors does not have a major influence on either the size of the song nuclei or any of the cellular attributes that we measured. The correlation between song complexity and the size of song nuclei found in marsh wrens and other bird species is thus not primarily the outcome of differential song learning expericnces early in life. This observation does not, however, preclude the possibility that song learning and/or subsequent production have some subtle effects on development of the song nuclei. In a companion study using the other telencephalic lobe of these same brains, males in the large repertoire tutor group had a greater density of neuronal dendritic spines than the small rep- ertoire birds in HVC, but not in RA (Airey et al., 1994). In our study, the variance in the absolute volume of RA (but not HVC) was greater in the large repertoire group. Although this difference in variance could be an effect of differential learning, it could also reflect sampling error, given our relatively small sample sizes.

Song learning might have a more pronounced effect on the dcvclopment of the song nuclei if one were to tutor birds with extremely large repertoires and so induce them to function at their maximum learning capacity. Such an experiment would be an interesting extension of the present study. Note, however, that male wrens tutored with 200 song types learned to produce a median of 45 song types in the study of Kroodsma and Canady (1985), which suggests that we probably approached maximum learning in our large repertoire birds.

Our data are consistent with the developmental scenarios presented in the two alternative hypotheses presented earlier. Either birds endowed with large song nuclei are able to subsequently learn large numbers of song types (Hypothesis 2), or the size of song nuclei and of song repertoires develop independently and in parallel (Hypothesis 3). The correlation that we found between repertoire size and the volume of song nuclei within the group tutored with large repertoires (Fig. 1) is consistent with both of these ideas.

How might one discriminate experimentally between the two alternative hypotheses? As a first step, one could compare the development of song nuclei in different individuals of a species at different stages of song learning. A prediction of Hypothesis 2 might be that individual differences in the size of developing song nuclei should appear by the early stages of song learning. According to Hypothesis 3, however, individual differences in the size of song nuclei may not appear until later stages of song learning, when behavioral differences in song production may also first become evident. Such a study has yet to be done.

The present study suggests that birds can develop song nuclei of normal size in the absence of normal song learning experience. Other support for this general conclusion comes from the finding that deafening young male zebra finches does not alter the normal developmental time course of changes that occur in ncuron number in the song nuclei IIVC and Area X (Burek et al., 1991). What factors, then, might guide the development of these nuclei so as to result in individual differences in brain space devoted to song? One factor may be genetic variation between individuals, which could affect such processes as the rates of neurogenesis and programmed cell death at critical times of development.

A second factor might be individual differences in epigenetic influences, such as circulating concentrations and/or metabolism of steroid hormones. Gonadal steroids and their metabolites play an important role in the organization of the song control pathways and in song learning behavior (for reviews, see Arnold, 1992; Doupe, 1993; Brenowitz and Kroodsma, in press). Steroids can be derived from the bird's own gonads and brain metabolism (Schlinger and Arnold, 1993), and, before hatching, from the yolk of the egg (Schwabl, 1993). Individual differences in stcroid concentrations available at critical stages could start development of the song control circuits down different ontogenetic pathways, so that some young birds develop larger song nuclei than others, which may then enable them to learn larger song repertoires.

A third potential source of individual differences in development of the song system is environmental factors. The amount 
of yolk invested in an egg, and the amount and quality of food given to individual chicks after hatching, for example, might influence the development of song nuclei. The contribution, if any, of such environmental factors to ontogeny of the song system is unknown.

We have demonstrated that song learning experience does not play a dominant role in determining the amount of brain space devoted to song in an individual bird. It would also be productive to investigate the roles that genetic, epigenetic, and environmental factors play in the development of the song control system. The development of neural systems in general depends on an interaction between genetic, epigenetic, and environmental factors (Purves and Lichtman, 1985). Similar considerations can be invoked to explore differences between closely related species in the amount of brain space devoted to song behavior.

\section{References}

Airey DC, DeVoogd TJ, Kroodsma DE (1994) Morphology of song control neurons in differentially tutored eastern marsh wrens. Soc Neurosci Abstr 20:164.

Arnold AP (1992) Developmental plasticity in neural circuits controlling birdsong: sexual differentiation and the neural basis of learning. J Neurobiol 23:1506-1528.

Railey $\mathrm{CH}$, Kandel FR (1993) Structural changes accompanying memory storage. Annu Rev Physiol 55:397-426.

Brenowitz EA, Arnold AP (1986) Interspecific comparisons of the size of neural song control regions and song complexity in duetting birds: evolutionary implications. J Neurosci 6:2875-2879.

Brenowitz E, Kroodsma DE (in press) The neuroethology of bird song. In: Ecology and evolution of acoustic communication in birds (Kroodsma DE, Miller DH, eds), in press. Ithaca, NY: Cornell UP.

Brenowitz E, Nalls B, Kroodsma DE, Horning C (1993) Female marsh wrens do not provide evidence of anatomical specializations of song nuclei for perception of male song. J Neurobiol 25:197-208.

Burek MJ, Nordeen KW, Nordeen EJ (1991) Neuron loss and addition in developing zebra finch song nuclei are independent of auditory experience during song learning. J Neurobiol 22:215-223.

Cammermeyer J (1967) Artifactual displacement of neuronal nucleoli in paraffin sections. J Hirnforsch 9:209-224.

Canady R, Kroodsma DE, Nottebohm F (1984) Population differences in complexity of a learned skill are correlated with the brain space involved. Proc Natl Acad Sci USA 81:6232-6234.

DeVoogd TJ, Krebs JR, Healy SD, Purvis A (1993) Relations between song repertoire size and the volume of brain nuclei related to song: comparative evolutionary analyses amongst oscine birds. Proc R Soc Lond [Biol] 254:75-82.

Doupe AJ (1993) A neural circuit specialized for vocal learning. Curr Biol 3:104-111.

Konigsmark BW (1970) Methods for the counting of neurons. In: Contemporary research methods in neuroanatomy (Nauta WSH, Ebbesson SOE, eds), pp 315-340. New York: Springer.

Kroodsma DE (1979) Vocal dueling among male marsh wrens: evidence for ritualized expressions of dominance/subordinance. Auk 98: 506-515.

Kroodsma DE, Canady RA (1985) Differences in repertoire size, singing behavior, and associated neuroanatomy among marsh wren populations have a genetic basis. Auk 102:439-446.

Kroodsma DE, Pickert R (1980) Environmentally dependent sensitive periods for avian vocal learning. Nature 288:477-479.

Lanyon WE (19/9) Development of song in the wood thrush (Hylocichla mustelina), with notes on a technique for hand-rearing passerine birds from the egg. Am Mus Novitates No. 2666.

Nottebohm F (1981) A brain for all seasons: cyclical anatomical changes in song control nuclei of the canary brain. Science 214: $1368-1370$.

Nottebohm F, Kasparian S, Pandazis C (1981) Brain space for a learned task. Brain Res 213:99-109.

Purves D. Lichtman JW (1985) Principles of neural development. Sunderland, MA: Sinauer.

Schlinger BA, Arnold AP (1993) Estrogen synthesis in vivo in the adult zebra finch: additional evidence that circulating estrogens can originate in the brain. Endocrinology 133:2610-2616.

Schwabl H (1993) Yolk is a source of maternal testosterone for developing birds. Proc Natl Acad Sci LTSA 90:11446-11450.

Simpson HB, Vicario DS (1991) Early estrogen treatment alone causes female zebra finches to produce learned, male-like vocalizations. J Neurobiol 22:755-776. 